\title{
New prototype neuronavigation system based on preoperative imaging and intraoperative freehand ultrasound: system description and validation
}

\author{
Laurence Mercier - Rolando F. Del Maestro - Kevin Petrecca - Anna Kochanowska • \\ Simon Drouin - Charles X. B. Yan - Andrew L. Janke • \\ Sean Jy-Shyang Chen • D. Louis Collins
}

Received: 8 April 2010 / Accepted: 13 September 2010

(c) CARS 2010

\begin{abstract}
Purpose The aim of this report is to present IBIS (Interactive Brain Imaging System) NeuroNav, a new prototype neuronavigation system that has been developed in our research laboratory over the past decade that uses tracked intraoperative ultrasound to address surgical navigation issues related to brain shift. The unique feature of the system is its ability, when needed, to improve the initial patient-to-preoperative image alignment based on the intraoperative ultrasound data. Parts of IBIS Neuronav source code are now publicly available on-line.

Methods Four aspects of the system are characterized in this paper: the ultrasound probe calibration, the temporal calibration, the patient-to-image registration and the MRI-ultrasound registration. In order to characterize its real clinical precision and accuracy, the system was tested in a series of adult brain tumor cases.

Results Three metrics were computed to evaluate the precision and accuracy of the ultrasound calibration. 1) Reproducibility: $1.77 \mathrm{~mm}$ and $1.65 \mathrm{~mm}$ for the bottom corners of the ultrasound image, 2) point reconstruction precision $0.62-0.90 \mathrm{~mm}$ : and 3) point reconstruction accuracy: $0.49-0.74 \mathrm{~mm}$. The temporal calibration error was estimated
\end{abstract}

L. Mercier $(\varangle) \cdot$ A. Kochanowska · S. Drouin · C. X. B. Yan ·

S. J.-S. Chen · D. L. Collins

McConnell Brain Imaging Center, Montreal Neurological Institute,

McGill University, Montreal, QC, Canada

e-mail: laurence@bic.mni.mcgill.ca

R. F. Del Maestro · K. Petrecca

Brain Tumour Research Centre, Montreal Neurological Institute and Hospital, McGill University, Montreal,

QC, Canada

A. L. Janke

Department of Geriatric Medicine, The Australian National

University, Canberra, SA, Australia to be $0.82 \mathrm{~ms}$. The mean fiducial registration error (FRE) of the homologous-point-based patient-to-MRI registration for our clinical data is $4.9 \pm 1.1 \mathrm{~mm}$. After the skin landmarkbased registration, the mean misalignment between the ultrasound and MR images in the tumor region is $6.1 \pm 3.4 \mathrm{~mm}$. Conclusions The components and functionality of a new prototype system are described and its precision and accuracy evaluated. It was found to have an accuracy similar to other comparable systems in the literature.

Keywords Application accuracy . Image-guided surgery · Intraoperative imaging . Intraoperative ultrasound · Neuronavigation · Registration

\section{Introduction}

When resecting brain tumors, conventional neuronavigation systems based on preoperative computed tomography (CT) or magnetic resonance imaging (MRI) are limited by the fact that these images do not reflect the surgical reality of the patient throughout the whole procedure. The main goal of intraoperative imaging like ultrasonography or intraoperative MRI (iMRI) is precisely to address that limitation.

Intraoperative ultrasound

Intraoperative brain ultrasonography is not new. The first papers discussing the use of ultrasound to image the brain were published in 1947 [1] and 1950 [2-4] using one-dimensional A-mode images. The first papers using real-time B-mode 2D imaging as we know it today were published in the early eighties [5,6]. A new era in ultrasound imaging began with the advent of tracked ultrasound [7-11]. With this technology, the position and orientation of the ultrasound probe can 
be known in real-time. Since the ultrasound probe is tracked in 3D space, the $\mathrm{x}, \mathrm{y}, \mathrm{z}$ coordinate of each ultrasound image pixel is known and can be matched with multiplanar reformatted preoperative CT or MR images. The latest commercial neuronavigation systems like the VectorVision ${ }^{\circledR}{ }^{\circledR}$ [12] (BrainLAB, Heimstetten, Germany) and the StealthStation Treon ${ }^{\circledR}$ Treatment Guidance System (Medtronic, Louisville, CO, USA) have incorporated tracked ultrasound and enable acquisition and visualization of 2D ultrasound with the corresponding preoperative image data. In addition to this feature, the commercial SonoWand ${ }^{\circledR}$ system [13] (Mison, Trondheim, Norway) allows the reconstruction of 3D ultrasound volumes from $2 \mathrm{D}$ ultrasound slices in near real-time. These reconstructed intraoperative volumes can then be visualized with their corresponding preoperative $\mathrm{CT}$ or MR data sets. A number of research groups have also proposed similar 3D ultrasound visualization possibilities [14-16].

\section{IMRI/ultrasound}

Studies comparing iMRI and intraoperative tracked ultrasound are rare. Tronnier et al. [17] used a low-field iMRI system and a 3D ultrasound in parallel for seven tumor cases. They found the iMRI system bulky and that it interfered with many surgical instruments, including the microscope. Gerganov et al. [18] compared the SonoWand ${ }^{\circledR}$ system, which allows 3D ultrasound reconstructions, with a highfield iMRI system during brain tumor resection procedures. They found that the image quality before the resection to be of similar quality with both modalities. The advantage of ultrasound over iMRI was its significantly shorter preparation and acquisition time and its drawback, the limited field of view. The field of view is a limitation of systems using optical tracking, because in these systems the probe must be seen by the tracking camera at all times thereby limiting the freedom of motion. However, tracked ultrasound costs a fraction of the price of an iMRI system.

IMRI and ultrasound have both been extensively used in brain tumor surgery, both to visualize the tumor and its boundaries before surgery, and to assess residual tumor toward the end of the resection. Even for experienced neurosurgeons, it can be difficult to define tumor margins [19], and intraoperative imaging has been found to be helpful in that regard. Unsgaard et al. [20] have shown that in 53\% of cases in which the resection was otherwise considered complete, 3D ultrasound allowed them to identify residual tumor. Knauth et al. [21] reported similar findings using a 0.2 Tesla iMRI system in a series of 38 high-grade glioma patients. Recently, Hatiboglu et al. [22] found that their high-field iMRI helped finding residual tumor in $47 \%$ of their glioma cases. When comparing both modalities, Tronnier et al. [17] found thought iMRI was superior to ultrasound in terms of residual tumor evaluation at the end of surgery.
In terms of visualizing tumors before the resection, van Velthoven et al. [23] have shown that ultrasound is as reliable as MRI to delineate gliomas, metastases and meningiomas. It may even be superior to MRI in defining low-grade tumor boundaries [24]. Van Velthoven et al. [23] also found that ultrasound enabled the differentiation of more structural details within the tumor. They also found that ultrasound could more distinctively delineate between the cystic and the solid part of a tumor compared to MRI.

Another advantage of ultrasound over MRI is the intuitive nature of the signal intensity, which measures the differences in stiffness and density between tissues. In tumor cases for example, neurosurgeons often use the haptic feedback from the instruments to help them differentiate between normal and pathological tissue when they are visually very similar. In these cases, the ultrasound signal provides the surgeon with information similar to what their sense of touch is providing.

\section{Brain shift}

Intraoperative imaging can also be a valuable tool for characterization and correction of the well-known brain shift problem. After opening the dura, for a brain tumor patient, the brain shifts because of cerebrospinal fluid drainage or leaks, tissue resection, swelling, cyst drainage, etc. The main cause of brain shift is often the tumor resection itself. By removing tumor tissue and possibly draining associated cysts, the brain tissue around the tumors changes considerably in shape. If the tumor is deep, creation of a resection channel also impacts on the brain. When retractors are used, the brain obviously deforms. Furthermore, retractors' compressing action can make the surrounding brain swell by interfering with the venous drainage. However, brain shift can be significant even before the resection starts [25]. This is particularly true if the preoperative scans are done days before the operation. Within only a few days, an aggressive tumor has time to grow and the effect of steroids used to reduce edema can lead to important changes in the brain's morphology. Gravity plays a role throughout the operation as well and displaces each lobes of the brain differently [26], although its effects are minimized when the patient is operated in the same head position as in the scanner (supine).

Brain shift during brain tumor surgery is a complex phenomenon that has been studied by several groups, often with the help of iMRI [26-31]. Only one will be detailed here for their very thorough investigation. Nabavi et al. [26] have imaged 25 tumor patients at four time points during surgery, in an open-bore 0.5T iMRI scanner. As most groups, they found the brain surface sunk with gravity, even well after dural opening. In their study, the maximal surface shifts ranged from almost no detectable shift for the smaller lesions to up to $5 \mathrm{~cm}$ for the larger lesions. The mean maximal 
shift was found for larger tumors right after the resection, but before dural closure.

New prototype neuronavigation system

The aim of this report is to present a new prototype neuronavigation system that has been developed in our research laboratory over the past decade to address the brain shift issue identified above. The system continues previous work published by our research group in 1998 by Comeau et al. [32]. The system is called IBIS NeuroNav; IBIS stands for Interactive Brain Imaging System. The system is interactive in the sense that the surgeon can interact with the data to visualize different structures, examine spatial relationships, acquire intraoperative ultrasound data and use it to (re-) register preoperative images. The purpose of developing our own navigation system was to have a robust software platform that would facilitate integration of different research prototype tools into a system that could be tested and validated in real clinical conditions, while also benefiting the practice of the neurosurgeons at our institution. Our goal is ultimately to design a neuronavigation system with improved navigation accuracy to allow an optimal surgery.

The system allows planning and navigation on preoperative medical image data (MRI, CT, functional MRI (fMRI), positron emission tomography (PET), etc.) as well as navigation with intraoperative tracked ultrasound. It also incorporates a very broad range of functionalities, including ultrasound probe calibration, automatic 3D surface segmentations, 3D ultrasound reconstruction from tracked 2D ultrasound images and advanced inter-modality registration techniques. In addition, it allows automatic intraoperative correction of the patient-to-preoperative scan misalignment based on intraoperative ultrasound data, which to our knowledge is the first system to do so.

The following sections describe the components, functionalities and characterization of the system. In order to characterize its real clinical precision and accuracy, the system was tested in a series of adult brain tumor cases. Four aspects of the system are characterized in this paper: the ultrasound probe calibration, the temporal calibration, the patient-to-image registration and the MRI-ultrasound registration.

\section{System description}

Components of the system

IBIS NeuroNav is composed of a tracking system, an ultrasound scanner, and a workstation. Figure 1 shows a typical setup of the neuronavigation system when used in the operating room. Since IBIS NeuroNav is a research prototype,

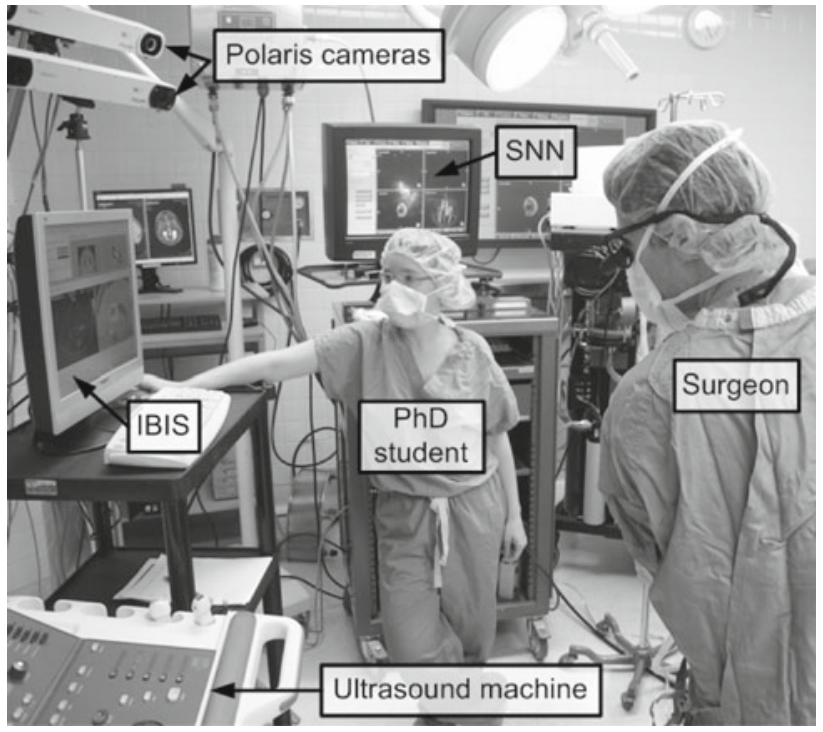

Fig. 1 Components of our neuronavigation system and layout of the equipment when using two neuronavigation systems (IBIS NeuroNav and $\mathrm{SNN}$ ) in parallel

it is always used in parallel with a commercial neuronavigation system. At the time of the study, the SNN system (Surgical Navigation Specialists Inc., Missisauga, Ontario, Canada) was used by the hospital (see Fig. 1). The following paragraphs detail the role and the relationships of each component in the system.

The workstation runs Debian GNU-Linux 3.1 and has a dual core Intel ${ }^{\circledR}$ Xeon ${ }^{\circledR} 3 \mathrm{GHz}$ processor. The screen is a 19 inch ViewSonic LCD monitor (ViewSonic Co, Walnut, CA, USA). The workstation is used to run the IBIS NeuroNav software and communicate with the tracking system and ultrasound device. The main design of the IBIS NeuroNav software was completed by the author D.L.C., and the individual components were designed and implemented by authors S.D. and A.K. IBIS NeuroNav is written in C++ and uses the Visualization ToolKit (VTK) libraries [33] for image processing and visualization and Qt 3 for the graphical user interface. IBIS NeuroNav reads and writes the medical image data in the MINC format [34], which is the format used at our institute for image processing. The MINC file format contains coordinate information that enables the images and volumes to be registered and compared to each other using tools from the publicly available MINC library (packages. bic.mni.mcgill.ca).

The ultrasound scanner enables intraoperative imaging of the patient during surgery. The ultrasound machine is an HDI 5000 (ATL/Philips, Bothell, WA, USA) and the 2D probe used is a P7-4 MHz phased array transducer. The ultrasound images are captured by IBIS NeuroNav through a Pinnacle PCTV ${ }^{\mathrm{TM}}$ frame-grabbing card (Pinnacle Systems, Inc., Mountain View, CA, USA). 


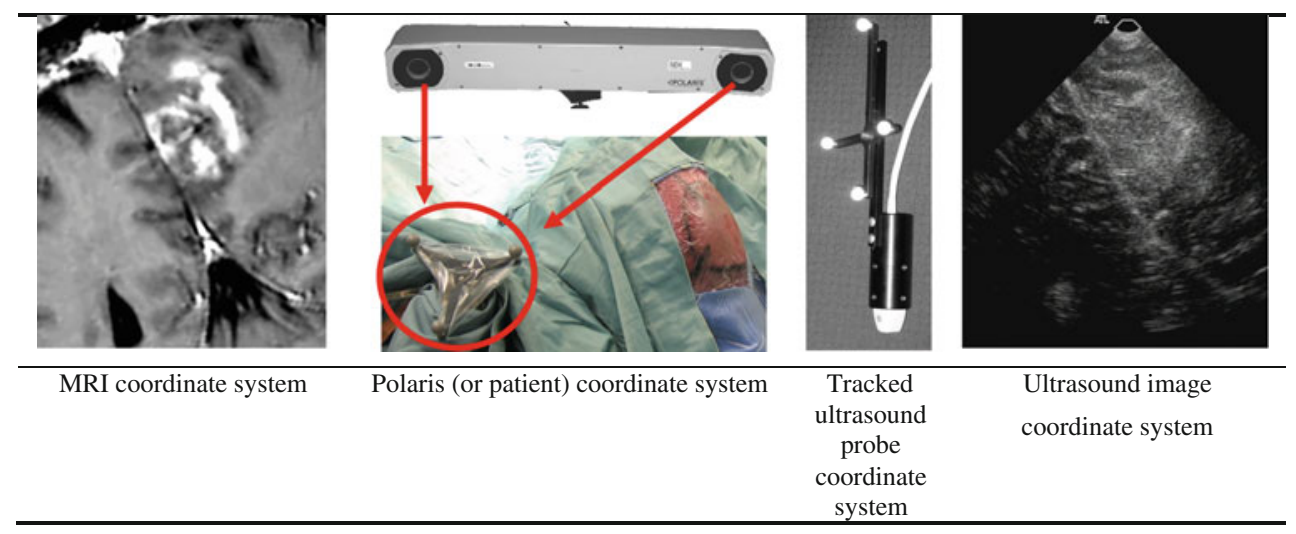

Fig. 2 During surgery, navigation with the pointer is done in the Polaris coordinate system (second column). In order to correlate the pointer's position with the MRI (first column) and ultrasound images (fourth column), they must also be transformed in that coordinate system. The MRI is transformed in the Polaris coordinate system through the

Tracking is done with a Polaris ${ }^{\circledR}$ infrared optical system (Northern Digital, Waterloo, Ontario, Canada).

The tracking system establishes a coordinate system in which the patient and tools can be tracked in 3D space. As seen in Fig. 1, each navigation systems (IBIS NeuroNav and SNN) is connected to their own optical tracking devices that are placed one above the other. IBIS NeuroNav can track any tools compatible with the Polaris tracking system. For simplicity, when used in the operating room, IBIS NeuroNav tracks the same tools as the SNN system: a reference (TA002, Traxtal Technologies Inc., Toronto, Ontario, Canada) and a pointer (Aesculap AG \& Co. KG, Tuttlingen, B-W, Germany). In addition, IBIS NeuroNav tracks a 2D ultrasound probe. The reference is rigidly attached to the Mayfield clamp which is firmly attached to the patient's head and defines the origin of the Polaris ${ }^{\circledR}$ coordinate space (see second column of Fig. 2). The pointer is tracked by the Polaris ${ }^{\circledR}$ camera and is used to navigate in this space. IBIS NeuroNav also tracks an ultrasound probe, on which reflective spheres are rigidly fixed using a TA003 tracker (Traxtal Technologies Inc., Toronto, Ontario, Canada). The TA003 is screwed on a custom-made anodized aluminum adapter, which consists of a rod attached to a cylinder (see third column of Fig. 2). The cylinder rigidly holds the probe in place with 8 screws. When used intraoperatively, the probe cannot be sterilized, so it is draped in a sterile plastic sleeve during surgery and sterile passive spheres are clipped through the bag.

Mapping the MRI and ultrasound images in Polaris space

To map a voxel from an MRI to its corresponding ultrasound pixel (right), three transformations are necessary. The MRI patient-to-image registration. The transformation from the tracked probe to the Polaris coordinate system is given by the Polaris camera directly. The ultrasound image is mapped in the tracked probe's coordinate system (third column) through a probe calibration procedure

must first be transformed to the Polaris coordinate system, second to the tracked ultrasound coordinate system and third to the ultrasound image coordinate system.

\section{MRI to Polaris space}

The transformation from MRI space to patient space (see Fig. 2), also called the patient-to-image registration, is done using seven anatomical landmarks including the nose (bridge), the eyes (bilateral inner and outer canthi) and the ears (bilateral tragus valleys). The patient-to-image registration is done simultaneously for SNN and IBIS NeuroNav, with both systems tracking the same reference and pointer. Both systems report a root mean square error (RMSE), also called the mean Fiducial Registration Error (FRE). In addition to the mean FRE, IBIS NeuroNav also computes the individual FRE for each landmark point pair, which can help improve the initial registration. The quality of the registration is evaluated by touching the scalp with the pointer while simultaneously verifying the probe-skin distance on the neuronavigation screen. If the registration is not satisfactory, points may be removed/added or redone. Once completed, the patient-to-image registration allows the surgeon to relate the patient's head to the preoperative scans.

\section{Ultrasound to Polaris space}

The transformation from the tracker to the Polaris space is given by the tracking system. Ultrasound probe calibration consists of finding the last transformation between the 2-D coordinates of pixels in the ultrasound image into the tracker space. Finding this transformation is mandatory for all systems using tracked ultrasound. For the calibration of our 

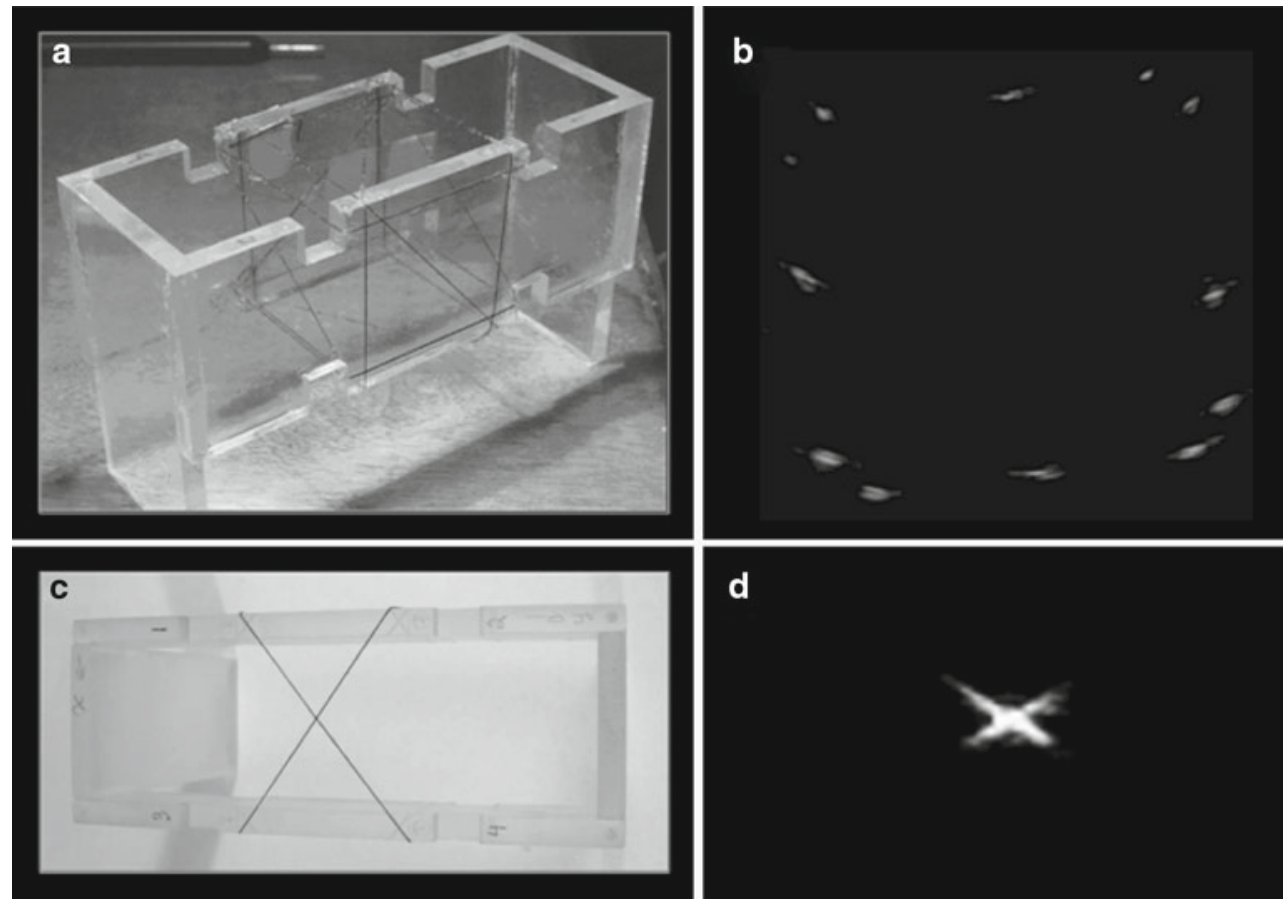

Fig. 3 Phantoms used for calibration and its validation. a 4 z-fiducials phantom with $\mathbf{b}$ a typical ultrasound image of the $z$-fiducial phantom. c Cross-wire phantom used for calibration validation with $\mathbf{d}$ a typical ultrasound image of the cross-wire phantom

ultrasound probe, a phantom with four z-fiducials [35] was used (see Fig. 3a). The wires in this phantom form $Z$ shapes that are intersected by the ultrasound image plane, and each of these z-fiducial produces three visible points on the image (see Fig. 3b). Using similar triangles, the position of the center point can be found and only that point is then considered as a homologous point between the image and phantom. A least squares minimization technique is used to minimize the distance between the two sets of points in order to find the unknown calibration parameters. There are 3 parameters to describe the translation, 3 for the rotation and 2 for the scaling. A detailed description of the calibration procedure and calibration phantoms can be found in Mercier et al. [36].

\section{Temporal calibration}

Temporal calibration is the process of synchronizing each ultrasound image with its appropriate pose coming from the tracking device. The temporal calibration of our system was evaluated using the technique defined in Treece et al. [37] with the StradX software [38]. The technique involves imaging the bottom of a tank filled with water by moving the probe up and down for a few seconds. The bottom of the tank produces a line that is automatically segmented from each ultrasound image. The temporal offset is found by computing the minimum root mean square error between the distances derived from the $b$-scans and those derived from the tracking device. Temporal calibration only needs to be done once and compensated for if necessary.

\section{System validation}

\section{Probe calibration validation}

To evaluate the calibration procedure, a second phantom with a simple cross-wire, (Fig. 3c, d) was used. To compensate for speed of sound distortions, the phantoms were imaged in water at room temperature mixed with $9.5 \%$ ethanol. Because the adapter and tracker are solidly screwed on the ultrasound probe, calibration does not need to be redone after each case.

The final calibration transformation (matrix) is generally obtained from a series of calibration matrices. Each of the individual calibration matrices leads to positioning the ultrasound plane at a slightly different location in world space. The stability or precision at which the image plane's position varies with each calibration trial is termed the calibration reproducibility. In this study, the calibration reproducibility was computed at 7 points in the ultrasound image.

Another common way of assessing the quality of the probe calibration is to image a phantom, ideally a phantom different from the one used for calibration, and evaluate the precision and accuracy at which points can be located on that phantom. For our experiments, the second phantom included a cross-wire (Fig. 3c, d) that was imaged from multiple viewing 
angles and two depths ( 65 and $95 \mathrm{~mm}$ ). The point of intersection of the cross was extracted from each ultrasound image and mapped in world space, forming a cloud of points. The tightness (or spread) of this cloud can be used to estimate the point reconstruction precision. Because the phantom was precisely built, the position of the wire-crossing is known in world space and is therefore considered as the gold standard value. The point reconstruction accuracy is obtained by comparing the position of each point in the cloud to this gold standard. As in other similar studies $[39,40]$, the mean Euclidian distance is reported here.

\section{Patient-to-image registration accuracy}

In order to evaluate the registration accuracy of each patientto-image registration, the mean FRE given by the neuronavigation systems was used. It represents the mean distance between corresponding fiducials after registration [41,42].

\section{MRI-ultrasound co-registration accuracy}

The magnitude of the MRI-ultrasound misalignment on these cases was evaluated by first estimating an ideal MRI-ultrasound registration as a baseline for comparison. As no gold standard is available for clinical data, our bronze standard [43] was defined by selecting corresponding anatomical landmarks in both modalities. For each case, a senior neurosurgeon (R.D.F.) chose seven to eleven corresponding anatomical features in both the ultrasound and MR volumes. The features included vessels, easily identifiable sulcal patterns, ventricle walls, etc. The mean absolute Euclidian distance between the corresponding points in both modalities was then computed to estimate the co-registration accuracy.

\section{Use of IBIS NeuroNav for brain tumor surgery}

\section{Patients}

As our laboratory is located in a neurological hospital, IBIS NeuroNav has been tested in the context of neurosurgery. Earlier prototypes of the system were tested clinically [44-46] on a few patients with various pathologies. Below, we describe the application of the current system to adult brain tumor resections on 14 patients. Tumor types consisted in seven high-grade gliomas, six low-grade gliomas and one dysembryoplastic neuroepithelial tumor (DNT). Tumor location was left frontal (7 cases), left temporal (5 cases), right parietal (1 case) and left occipital (1 case). All 14 cases could not be used for all accuracy metrics for different reasons. The results section clearly identifies how many patients were used for each metric and explains in each case why.
The following sections describe the processing and procedures that apply specifically to brain tumor cases, but the system may be used for many other types of interventions.

\section{Preoperative imaging and processing}

This section describes the preoperative preparation of the data as it was done at the time of this study (2006-2008). Note that during this first phase of development, the preoperative data preparations were not the focus of any research project in our laboratory. The clinical MR scanner at our institution is a 1.5 Tesla GE Signa EXCITE ${ }^{\circledR}$ (General Electric, Milwaukee, WI, USA). The standard preoperative MRI protocol for a tumor patient only includes one high resolution sequence, it is a global T1-weighted enhanced with double dose gadolinium $(\mathrm{TE}=8 \mathrm{~ms}, \mathrm{TR}=23 \mathrm{~ms}, \mathrm{FA}=$ $20^{\circ}, 0.5 \times 0.5 \mathrm{~mm}$ pixel size and $1-\mathrm{mm}$ slice thickness). Other sequences (T2/PD, T1 FLAIR, DWI) are also acquired, but with 5.5- $\mathrm{mm}$ slices; these are used only for diagnostic purposes. The high-resolution T1-weighted scan is the one used for navigation in most tumor cases. If a low-grade glioma is suspected, surgeons may request an additional higher-resolution $\mathrm{T} 2$-weighted scan $\left(\mathrm{TE}=92 \mathrm{~ms}, \mathrm{TR}=6.3 \mathrm{~s}, \mathrm{FA}=90^{\circ}\right)$ with a slice thickness of $2.2 \mathrm{~mm}$ that covers the tumor area. In tumors adjacent to eloquent cortex, the surgeon may request specific functional evaluation such as PET [47] and/or fMRI [48] studies. The fMRI data are acquired on a 3 Tesla Siemens Trio scanner (Magnetom TRIO; Siemens, Erlangen, Germany) using gradient echo EPI sequences. The fMRI protocol includes an anatomical T1-weighted MR scan which is rigidly registered to the preoperative T1-weighted image from the GE 1.5-Tesla scanner. The PET scans are acquired on an ECAT Exact HR+ scanner (Siemens/CTI, Knoxville, TN, USA). To map the PET functional activations on the preoperative MR scans, a rigid-body mutual informationbased registration is computed. Informed written consent was obtained from all patients according to the institutional guidelines established by the ethics committee of the Montreal Neurological Hospital and Institute.

The preoperative scans are first converted from DICOM into the MINC format [34]. Processing of these scans is done using the MINC library tools developed at our institution. The first step is to correct for intensity non-uniformities [49] in the images. Typically, the following 3D surfaces are then extracted from the MR volumes: skin, cortex, lateral ventricles, tumor(s) and selected parts of the vasculature (venous sinuses and vasculature near the tumor). All surfaces are extracted from the T1-weighted gadolinium-enhanced (gado) scan, the highest resolution scan. In cases of nonenhancing tumors, the 2.2- $\mathrm{mm}$ T2-weighted scan may be used for the tumor segmentation. The cortical surface is automatically extracted by calling a series of MINC tools. The main steps of the preprocessing are: 
Fig. 4 Example of 3D surface extractions for a left temporal brain tumor case. a Cortex in gray and vasculature in red b Vasculature in red, ventricles in blue and tumor in green c Ventricles in blue, tumor in translucent green and fMRI activation in pink $\mathbf{d}$ Ventricles in blue, tumor in translucent green, PET activations in gray and yellow
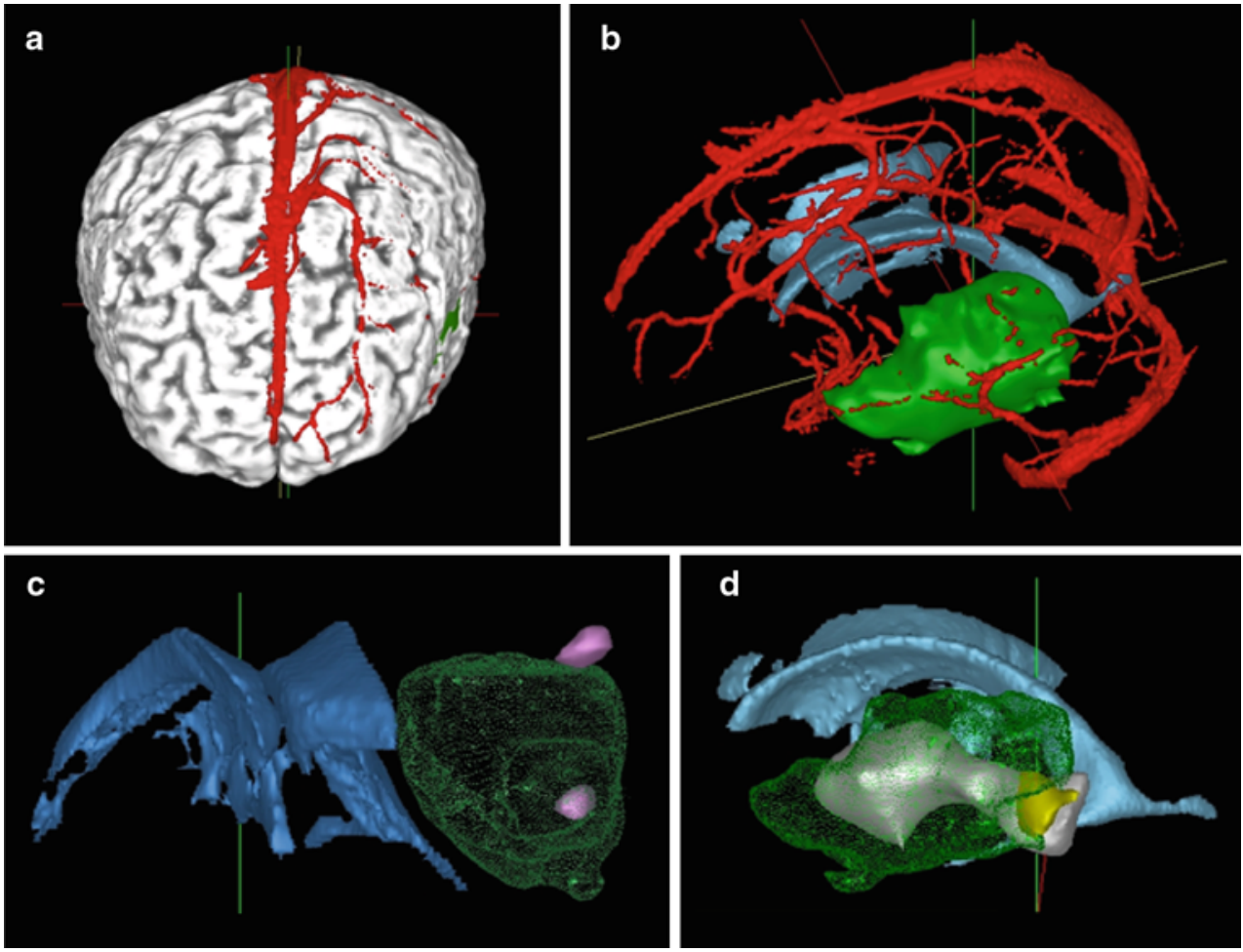

1) Map the scan into Talairach-like MNI space [50]

2) Classify tissues (gray-matter, white matter, cerebrospinal fluid) using an artificial neural network classifier [51]

3) Extract the cortical surface [52]

4) Color cortical surface according to curvature

5) Map the cortical surface back into native space

The other segmentations are done using a MINC tool called Display that allows simultaneous viewing and segmentation of volumes in standard coronal, sagittal and transverse cut planes as well as a 3D view. As a structure is manually labeled in any view, all other views are updated immediately. The skin surface is extracted using an iso-surface, with a threshold given by the user. Vessel segmentation is achieved with manual threshold-based techniques. The main veins and vessels around the tumor site are extracted. The tumor(s) and lateral ventricles are also extracted manually using thresholding. If functional data are available, the surgeon collaborates with a neuropsychologist to decide which activations are the most pertinent and at what value they should be thresholded. 3D surfaces of the selected functional areas are extracted in Display using iso-surfaces. The 3D surfaces are saved in a geometry file containing polygons which can be read and manipulated by the MINC tools as well as IBIS NeuroNav. Figures 4 and 5 show typical segmentations viewed in the IBIS NeuroNav software. The 3D surfaces are used for both planning and for neuronavigation by the surgeon. The coordinates of each object are derived from the initial MINC files, enabling them to be visualized and compared with the multimodal volumetric data.

Intraoperative ultrasound imaging

\section{Ultrasound image acquisition}

In our brain tumor series, ultrasound data were typically acquired three times during surgery. Each time, both conventional anatomical images (b-mode) and power Doppler images were usually acquired. Power Doppler was chosen over color Doppler for its greater sensitivity to vessels. A depth setting of $8 \mathrm{~cm}$ was generally chosen because it enables visualization of both the pathology and surrounding anatomical features like the falx or ventricles.

The first ultrasound acquisitions are always done on the dura, after the bone flap is removed, but before the resection begins. At this stage, the surgeon examines the B-mode acquisitions with their corresponding preoperative data in order to detect differences that could influence the surgical plan. In brain tumor cases, the comparison of this first ultrasound on dura to the preoperative MR volume is important since it allows the surgeon to assess the tumor characteristics on both modalities. Specifically, the surgeons examine the tumor boundaries using both modalities and if additional information concerning the tumor components (e.g. cyst, necrosis) can be extracted from the ultrasound. Since preoperative images may be carried out some time 


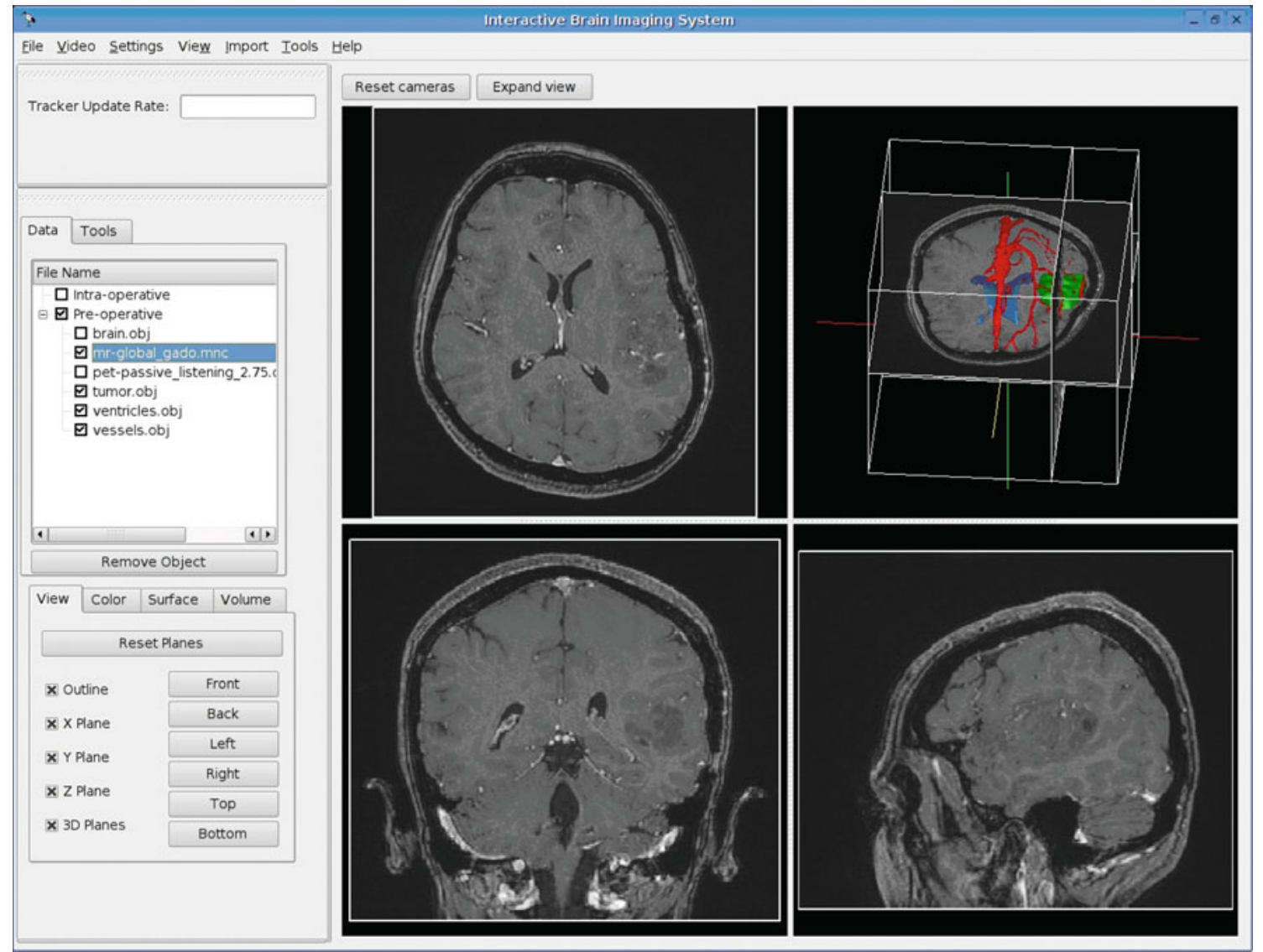

Fig. 5 Screenshot of IBIS NeuroNav showing an MR scan in the three usual orthogonal planes and the 3D view of these planes with the same segmented objects presented in Fig. 4. The case presented here is a patient with a low-grade left temporal glioma

before surgery, the ultrasound may detect changes in tumor and brain morphology since the preoperative scan. As magnetic resonance imaging angiograms are not part of our standard scanning protocol, the Doppler data allowed for a more detailed view of the vasculature adjacent or inside the tumor than the available MR scans. The second phase of acquisitions is done during the resection-either with the probe on the cortex or with the probe in the water-filled resection cavity. Such images enable the surgeon to evaluate the amount of resection completed. The last phase of acquisition is completed at the end of the resection to verify for possible residual tumor and bleeding.

While the ultrasound images are being acquired, the corresponding reformatted MR slice is displayed simultaneously, as seen in Fig. 6. Each ultrasound sweep is saved and can be viewed repeatedly. The ultrasound image can be superimposed on the MRI (see the "Blend" check box in the upper right corner of Fig. 6). The superposition allows a qualitative $2 \mathrm{D}$ evaluation of the misalignment between the two modalities. This double window with the blending option is a common feature of the most recent commercial neuronavigation systems. Because we think it is a particularly useful feature, we decided to implement it on our system as well. The position of the ultrasound plane relative to other segmented structures can also be viewed in the 3D window in real-time during the acquisition or during the playback (Fig. 7). Additional acquisition details are discussed in the clinical perspective section below.

\section{Ultrasound volume reconstruction}

In the literature, only a few systems can reconstruct the tracked 2D ultrasound into a 3D ultrasound volume $[13,14$, 53]. IBIS NeuroNav has this functionality (Fig. 8). It uses a pixel-based method [54], which means each pixel from each $2 \mathrm{D}$ image is inserted into a regular 3D grid that can be visualized and navigated like the preoperative data. Before reconstructing, the data are first masked: the real ultrasound signal is kept and everything else around it (e.g., annotations, scale bars) is masked to 0 (black). The reconstruction time depends on the size of the field of view (FOV), resolution and the number of frames that were acquired. The user can choose the desired resolution and interpolation method for the reconstruction. Typically, sweeps contain between 200 and 600 frames and are reconstructed at a voxel size of $0.3 \times 0.3 \times 0.3 \mathrm{~mm}$. For example, a sweep containing 425 


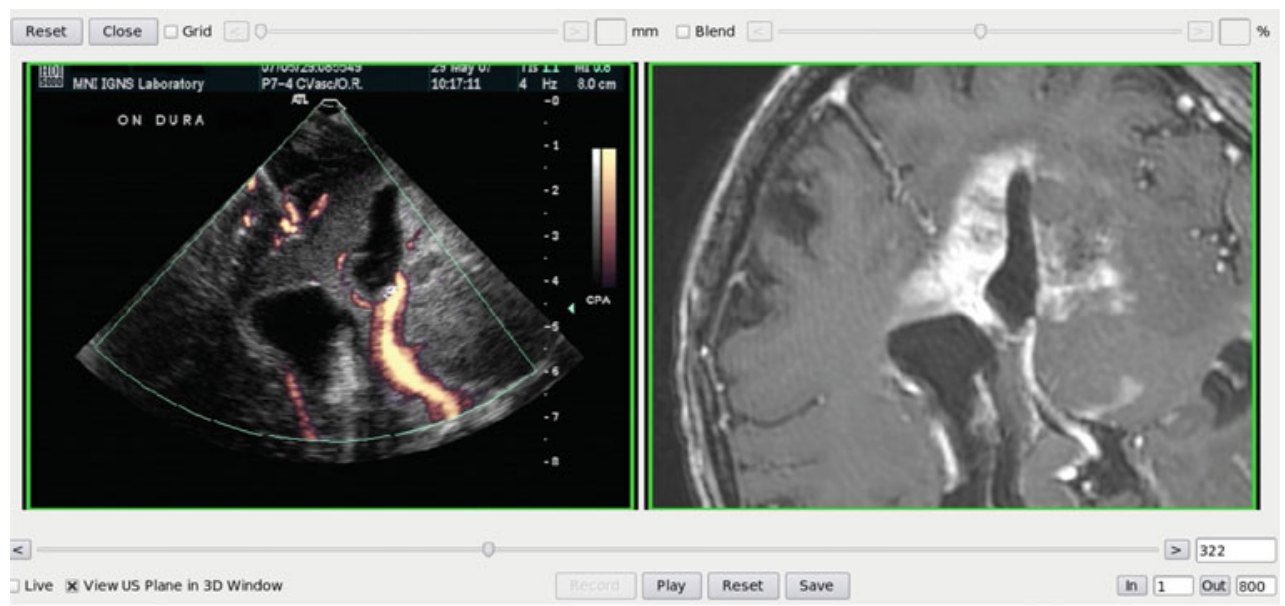

Fig. 6 When acquiring ultrasound images, the surgeon looks at this double window. The left side shows the current $2 \mathrm{D}$ ultrasound image (power Doppler ultrasound here) and the right side shows the

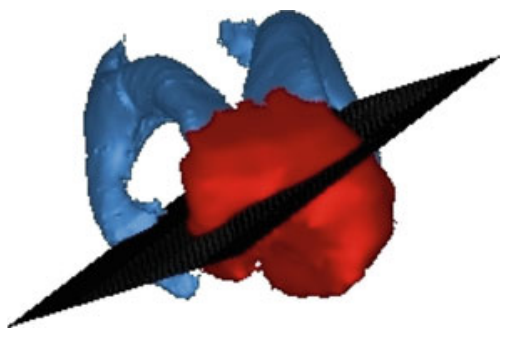

Fig. 7 Position of the ultrasound plane (in black) relative to a tumor (in red) and the lateral ventricles (in blue)

frames with a FOV of $19 \times 18 \times 11 \mathrm{~cm}$ takes about $10 \mathrm{~min}$ to reconstruct. Different reconstruction parameters can be chosen for the postoperative analysis of the data when the reconstruction time is less critical. For example, interpolation using a non-isotropic kernel, which better accounts for the ultrasound slice thickness, is possible. The ultrasound volumes are not segmented to extract $3 \mathrm{D}$ surfaces as for the preoperative scans.

Among the very useful features of our prototype system is the ability to compare the current ultrasound sweeps with previous acquisitions. The previous acquisition could be the preoperative data, or previous ultrasound data like that acquired on the dura. Such comparisons are very useful to better understand the ultrasound images acquired toward the end of surgery, as they that are not always easy to interpret.

\section{Intraoperative MRI-ultrasound realignment}

Even before the dura is opened, a mismatch of varying magnitude is usually observed between the tracked ultrasound images and reformatted preoperative images [53]. This mismatch is evidence of errors in the patient-to-image registration, inaccuracies in ultrasound probe calibration and corresponding slice of another volume, typically the preoperative T1-weighted MRI as it is shown here

potential brain shift described earlier. Since the intraoperative ultrasound data reflects the physical reality of the surgical field of interest, it can be used to recompute and refine the patient-to-image registration using intraoperative imaging. In neurosurgery, only a few authors have tried correcting the misalignment prospectively using ultrasound [55,56].

In the series of cases reported here, two automatic techniques for MRI-ultrasound rigid body registration were tested intraoperatively: a vessel-based technique $[57,58]$ and a method based on mutual information [59]. For each case, the best visual result was chosen for display to the surgeon. In the cases where the automatic registration is not satisfactory, the registration can be done manually by choosing corresponding landmarks in both modalities. Figure 9 shows an example of the MRI-ultrasound alignment before (left) and after (right) a manual rigid body correction.

\section{Results}

This section gives the numerical quantification of all the precision and accuracy tests done on the prototype system. The goal of this section is to establish what surgeons can expect when using the system clinically for neuronavigation.

\section{Ultrasound probe calibration evaluation}

Figure 10 shows the calibration reproducibility at seven locations in the 2D ultrasound image plane.

We imaged the cross-wire phantom from four different angles and with three different depth settings. Table 1 shows the point reconstruction precision and accuracy as well as the bias for these three settings. The distribution of the distancebased probe calibration results in Table 1 is more similar to 

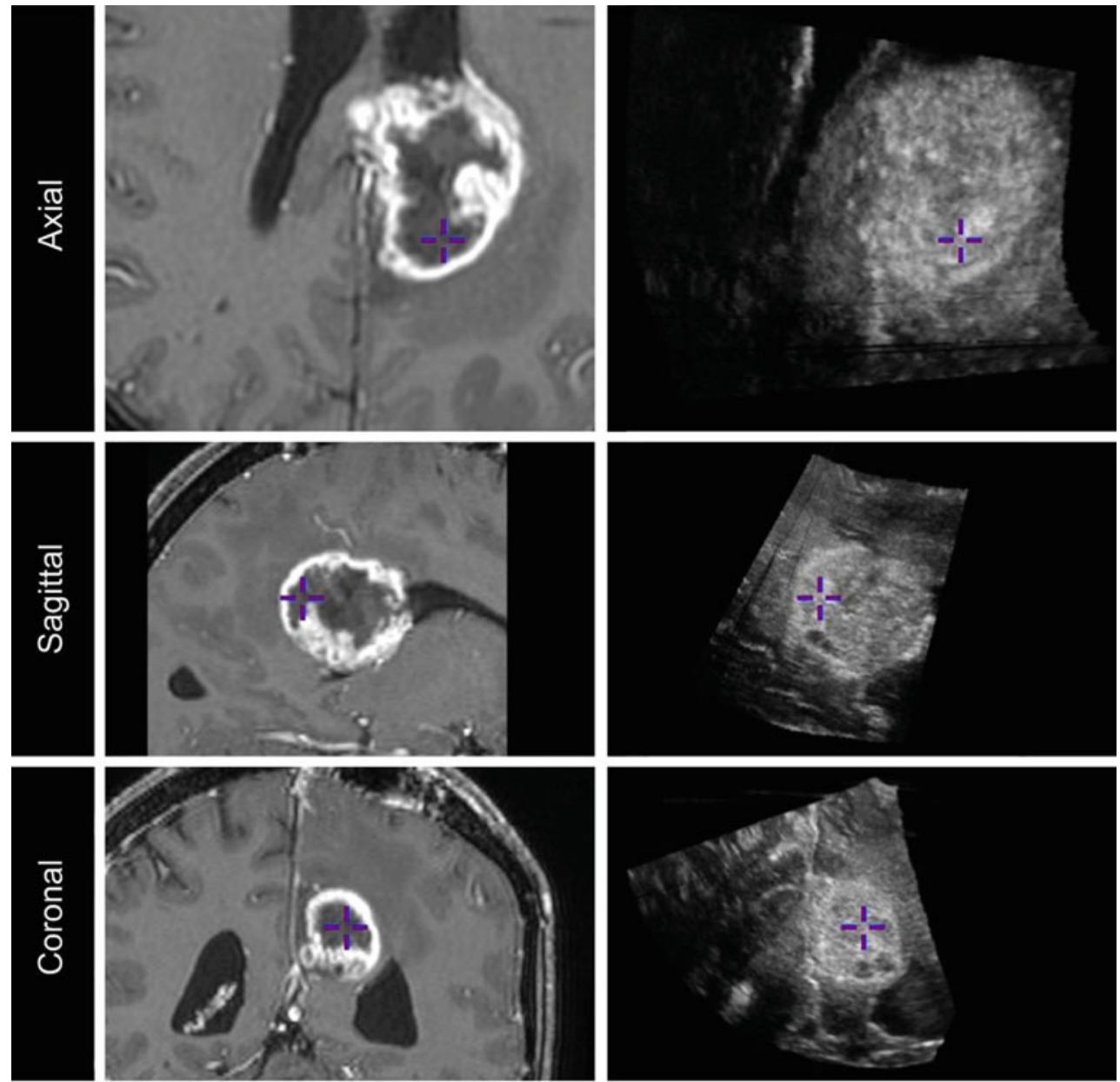

T1-weighted MR with gado

\section{D ultrasound}

Fig. 8 Axial, sagittal and coronal views of a gadolinium-enhanced T1-weighted MRI on the left with the corresponding slices from the reconstructed 3D ultrasound on the right
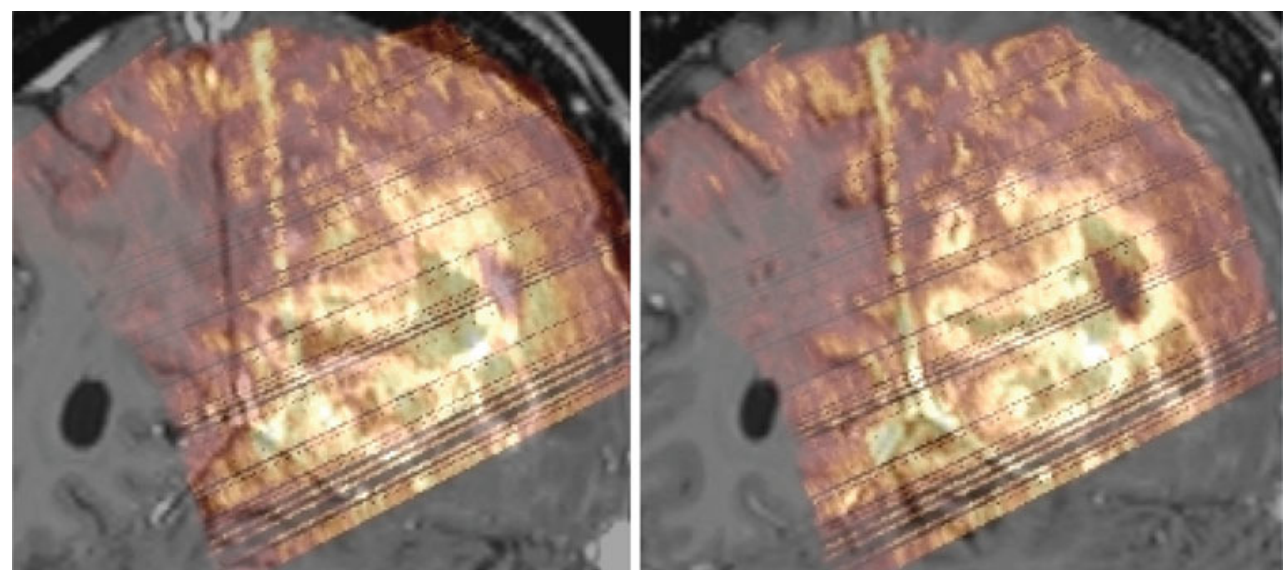

Fig. 9 2D reslice of a 3D ultrasound (in orange tones) overlaid on the corresponding MRI (in grayscale). Left initial misalignment (largest of the series), we see that the falx are very far one from another. Right after a manual rigid body correction of the alignment the falx now overlap 


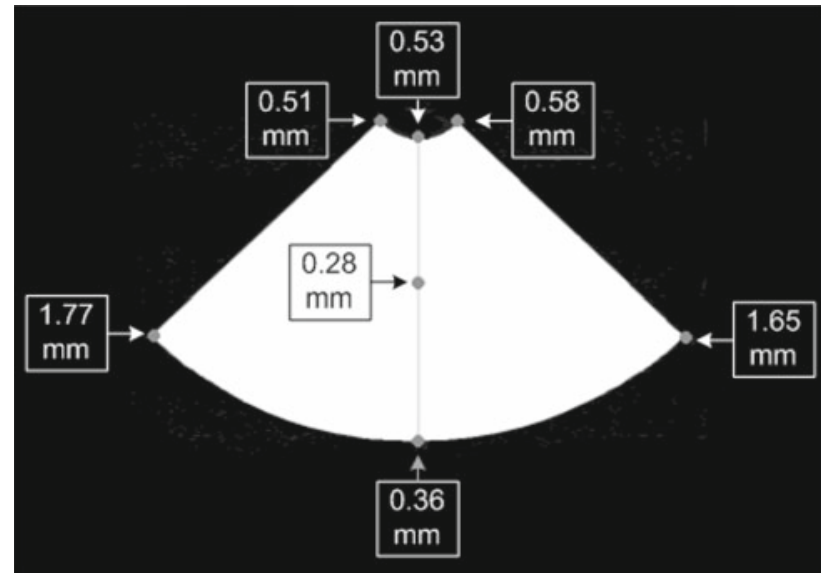

Fig. 10 Calibration reproducibility results at 7 locations in the ultrasound image plane

a Maxwell-Boltzmann distribution than a Gaussian distribution. However, we have decided to report the mean for an easier comparison with the four similar studies included in the discussion section.

Figure 11 shows 3D renderings of the reconstructed points (in green) for the third depth setting (depth setting $=95 \mathrm{~mm}$ with the point at $25 \mathrm{~mm}$ ).

\section{Temporal calibration evaluation}

The time lag between the Polaris ${ }^{\circledR}$ positions and the ultrasound images was estimated to be $82 \mathrm{~ms}$.

Patient-to-image registration evaluation

Problems with patient-to-image registration occurred on 2 of the 14 cases. In the first case of the series, the system was brought in when the patient was already draped and a very approximate registration was done using four cortical points. A software malfunction occurred in the second case. Based on the 12 remaining brain tumor cases using the skin fiducials method described earlier, the mean FRE value for IBIS NeuroNav is estimated to be $4.9 \pm 1.1 \mathrm{~mm}$. When recorded for the last 6 cases, the FRE value given by the SNN system used in parallel was almost identical $(4.6 \pm 1.2 \mathrm{~mm})$. As previously mentioned, each system had its own independent Polaris camera for tracking. A paired Student's t-test performed on both sets of FRE values, comparing the SNN and IBIS NeuroNav systems, showed no significant differences $(P=0.21)$.

\section{MRI-ultrasound co-registration accuracy}

In the 12 subjects with successful patient-to-image registration, three cases lacked B-mode imaging acquired on the dura. Therefore, the remaining 9 cases were used for quantitative validation. After the skin landmark-based registration, the mean MRI-ultrasound misalignment $(N=9)$ is $6.1 \mathrm{~mm}$ with values ranging from 2.4 to $10.9 \mathrm{~mm}$. In this series, the surgeons found that a correction of the initial MRI-ultrasound alignment was necessary in five out of the nine cases. Visually, the surgeons found that a rigid registration was sufficient to obtain a good alignment between the ultrasound taken on the dura and the preoperative MRI on these cases.

\section{Clinical perspective}

Two neurosurgeons were involved in testing and in the evaluation of system (R.F.D and K.P.). As Lindner et al. [60] reported, we found that the more experienced the surgeons are with ultrasound, the more useful they find it. Even if ultrasonography is widely available, neurosurgeons are generally less comfortable interpreting ultrasound compared to MR images. The surgeons involved in this project preferred looking at the double window (Fig. 6), which showed the 2D ultrasound slice with its corresponding MR reslice, over looking at the 2D ultrasound alone, as reported by Miller et al. [61]. They also found the 3D ultrasound reconstructions to be a very useful complement to the tracked 2D
Table 1 Point reconstruction precision, accuracy and bias for different depth settings

\begin{tabular}{|c|c|c|c|c|}
\hline $\begin{array}{l}\text { Depth setting } \\
(\mathrm{mm})\end{array}$ & $\begin{array}{l}\text { Depth of the image } \\
\text { point }(\mathrm{mm})\end{array}$ & $\begin{array}{l}\text { Reconstruction } \\
\text { precision }(\mathrm{mm})\end{array}$ & $\begin{array}{l}\text { Reconstruction } \\
\text { accuracy }(\mathrm{mm})\end{array}$ & $\begin{array}{l}\text { Bias } \\
(\mathrm{mm})\end{array}$ \\
\hline 65 & 25 & 0.90 & 0.74 & $\begin{array}{l}\mathrm{x}: 0.10 \\
\mathrm{y}:-0.25 \\
\mathrm{z}:-0.19\end{array}$ \\
\hline 65 & 45 & 0.62 & 0.49 & $\begin{array}{l}\mathrm{x}:-0.01 \\
\mathrm{y}: 0.01 \\
\mathrm{z}:-0.01\end{array}$ \\
\hline 95 & 25 & 0.62 & 0.82 & $\begin{array}{l}\mathrm{x}:-0.46 \\
\mathrm{y}:-0.02 \\
\mathrm{z}:-0.60\end{array}$ \\
\hline
\end{tabular}




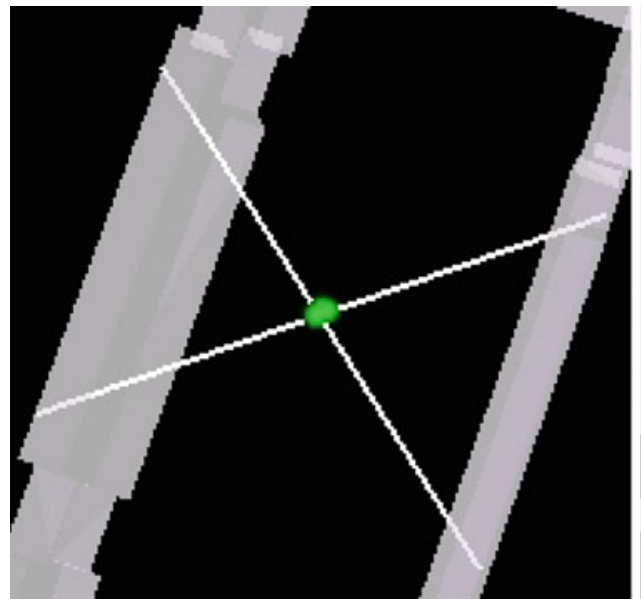

Fig. $113 D$ rendering illustrating the point reconstruction precision, accuracy and bias on a 3D model of the cross-wire phantom. In an ideal calibration, all four green points would be exactly located on the wire

ultrasound displayed in the double window with the MRI. The most obvious advantage of 3D over 2D ultrasound is the improved visualization of the 3D spatial relationships between brain structures-especially when compared to standard view (e.g., coronal, sagittal and axial) MR images. The $3 \mathrm{D}$ reconstructions also enable the production of $2 \mathrm{D}$ cross-sectional images at any angle, including angles impossible to achieve during the sweep.

\section{Discussion}

Ultrasound probe calibration evaluation

Comparing the ultrasound calibration quality with other publications is not straightforward since it depends on many factors such as the depth settings, tracking technology, range of viewing angles used and probe type. Therefore, the following comparisons must be considered with care.

The position of the bottom corners of the ultrasound image generally gives the largest calibration reproducibility values and for that reason many authors report only these measures. Blackall et al. [40] evaluated the CR on two different types of phantoms yielding 1.05 and $1.84 \mathrm{~mm}$. Prager et al. [62] computed the CR on four different phantoms and their values range from 0.92 to $5.37 \mathrm{~mm}$. Kowal et al. [63] measured the $\mathrm{CR}$ for four different types of calibration phantoms and obtained values ranging from 1.9 to $3.2 \mathrm{~mm}$. With a range of $0.28-1.77 \mathrm{~mm}$, our results are consistent with those published in the literature.

The point reconstruction precision was evaluated by Kowal et al. [63] on four phantoms. They obtained values ranging from 2.2 to $2.5 \mathrm{~mm}$. Prager et al. [62] also estimated the PRP value on four phantoms and obtained values ranging

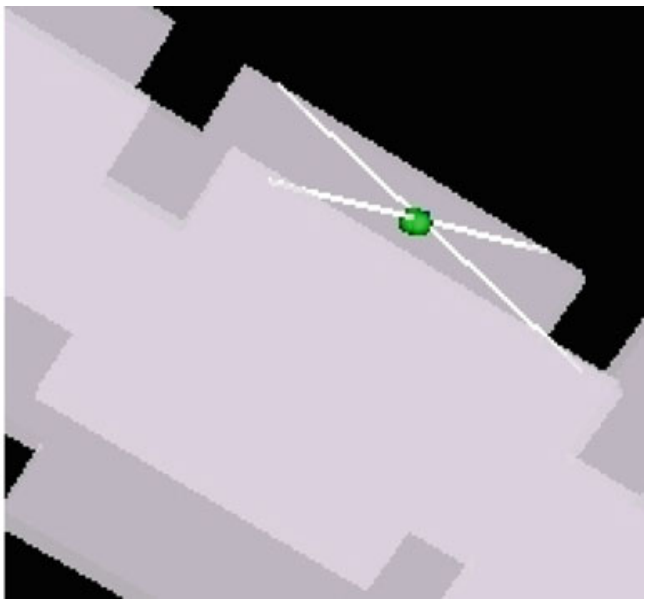

crossing. The rendering shows how the current calibration tends toward the ideal position

Table 2 Average distance and average velocity of one randomly selected sweep acquired by each surgeon

\begin{tabular}{llll}
\hline & $\begin{array}{l}\text { Number of } \\
\text { frames (N) }\end{array}$ & $\begin{array}{l}\text { Average distance } \\
\text { between 2 frames }(\mathrm{mm})\end{array}$ & $\begin{array}{l}\text { Average velocity } \\
(\mathrm{mm} / \mathrm{s})\end{array}$ \\
\hline Surgeon \#1 & 413 & 0.12 & 3.26 \\
Surgeon \#2 & 330 & 0.09 & 2.71 \\
\hline
\end{tabular}

from 1.65 to $3.43 \mathrm{~mm}$. Blackall et al. [40] used two phantoms and found PRP values of 0.80 and $1.15 \mathrm{~mm}$. At $0.71 \mathrm{~mm}$, our PRP is smaller than that previously reported in the literature.

Lindseth et al. [3] computed the point reconstruction accuracy (PRA) for three different phantoms and two different probes. Their PRA values range from 0.38 to $0.63 \mathrm{~mm}$. Blackall et al. [40] estimated the PRA using two phantoms at 1.15 and $1.16 \mathrm{~mm}$. Here, at $0.64 \mathrm{~mm}$, the PRA of IBIS NeuroNav is similar to those reported by Lindseth et al. and smaller than those reported by Blackall et al.

When looking at the bias results in Table 1 and Fig. 10, we found that the reconstructed points were approximately equally distributed around their ideal position and hence it was not necessary to correct for any bias in the calibration.

In summary, when all the calibration results are combined, we conclude that the quality of our ultrasound probe calibration is very similar to that obtained in the literature.

\section{Temporal calibration evaluation}

At $82 \mathrm{~ms}$, our latency was considered to be negligible and no compensatory action was taken. This decision was based on the findings by Rousseau et al. [64] and on the following reasoning. The average velocity and average distance between two frames were computed on a random sweep of $\mathrm{N}$ frames acquired by each surgeon (see Table 2). 
Based on these results, an approximate velocity of $3 \mathrm{~mm} / \mathrm{s}$ will be used in this example. With a latency of $82 \mathrm{~ms}$, the positioning error of a slice would be approximately $0.082 \mathrm{~s} * 3 \mathrm{~mm} / \mathrm{s}=0.246 \mathrm{~mm}$, which is lower than the point reconstruction accuracy of $0.64 \mathrm{~mm}$.

\section{Patient-to-image registration evaluation}

Comparing our surgical in vivo mean, FRE value with other publications is also difficult since it depends on numerous factors including the tracking mode; the preoperative imaging; the type, number and location of the markers, etc. Three comparable in vivo experiments using anatomical landmarks, optical tracking and similar preoperative MR imaging are presented here [65-67]. These studies report FRE values ranging from 2.3 to 3.2 , which is slightly lower than our estimated FRE of $4.9 \mathrm{~mm}$. Because the SNN systems yield almost identical FRE values as our system using a different software and a different Polaris ${ }^{\circledR}$ camera, these two components can be ruled out as a possible sources of error. On both the SNN and IBIS NeuroNav systems, the landmarks are chosen by an experienced technician on the gadoliniumenhanced T1-weighted MR scan and by the neurosurgeon or resident on the patient. Potential discrepancies could come from the misinterpretation of the location of these points since they were not chosen by the same person. Another significant source of inaccuracy might come from both the reference and the pointer tools. The tracking is done using old non-sterile spheres that may have been manipulated countless times. Over time, the tools may also have changed from their ideal geometry. In addition, pointer calibration is not done for each case.

Although no systematic quantitative measures were done, the FRE values did not seem to correlate with the navigation accuracy. In other words, even when the patient-to-image registration error was large, the tumors on ultrasound and MRI were sometimes very well aligned. This is in agreement with most authors in the literature, who did not find a correlation between the TRE and FRE [56,65,68-70]. Both Fitzpatrick et al. [71] and Shamir [72] actually recently proved that the FRE and TRE are in fact uncorrelated. Fitzpatrick et al. [73] define the FRE value as "the root mean square error in the fit of the fiducials after registration" and the TRE as "the true accepted measure of the accuracy of the registration process", which in other words is the registration error at a specific point of interest that was not incorporated in the registration computation.

MRI-ultrasound co-registration accuracy

The first publication to quantify the MRI-ultrasound co-registration accuracy using intraoperative data is from
Lindseth et al. [25]. They manually translated (in x,y,z) the MRI to best match the ultrasound volume on 12 patients. They report a mismatch ranging from 1.0 to $8.7 \mathrm{~mm}$ with a mean of $5.33 \pm 2.43 \mathrm{~mm}$. Letteboer et al. [74] also registered 3D volumes using translations only, but the registration was done automatically using mutual information. They found that prior to opening the dura, the average shift was $3.0 \mathrm{~mm}$ parallel to the direction of gravity, with a maximum of $7.5 \mathrm{~mm}$, and $3.9 \mathrm{~mm}$ perpendicular to the direction of gravity, with a maximum of $8.2 \mathrm{~mm}$. These results are very similar to ours even if they do not include rotational errors, which would likely have increased the values they found. Keles et al. [75] have estimated the co-registration accuracy in 2D only, using anatomical structures that typically do not shift like the choroids plexus and falx and obtained a mean accuracy lower than $2 \mathrm{~mm}$. The highest misregistration error was found on the cortex, with a mean of $3.5 \mathrm{~mm}$. These values are probably lower in part because they were done in $2 \mathrm{D}$. In our previous clinical work, Reinertsen et al. [58] measured the co-registration accuracy on 5 patients using 10 manually selected landmarks from Doppler ultrasound on the dura and the preoperative MR angiography after registration and found a mean co-registration accuracy of $7.34 \pm 2.1 \mathrm{~mm}$ with values ranging from 4.68 to $9.70 \mathrm{~mm}$. Using a closest point projection distance (CPPD) analysis [76] on 6 brain tumor cases, Ji et al. [56] found an initial tumor boundary misalignment between ultrasound and preoperative MRI of $2.5 \pm 1.3 \mathrm{~mm}$. As expected, the TRE for registration in the clinical setting is larger than when using phantoms.

The numbers cited above represent the co-registration accuracy when calculated with the ultrasound taken on the dura, before the surgery. This misalignment has two causes: system registration errors and real brain shift. Before opening the dura, our opinion is that the combined errors from the patient-to-image registration and probe calibration are larger than the brain shift (prior to resection). However, if the preoperative scans are acquired more than a few days before surgery, the proportion of the error coming from the brain shift component may increase.

Neither the StealthStation Treon nor the VectorVision2 allows for re-registration based on the ultrasound data to address the MRI-ultrasound misalignment issue. The SonoWand ${ }^{\circledR}$ system does not allow correcting for the misregistration either, but enables the surgeon to continue the surgery navigating solely with reconstructed ultrasound. This last solution circumvents the brain shift problem by enabling navigation with updated 3D ultrasound images. This solution is only attractive for surgeons that are comfortable looking at the anatomy solely with ultrasound, which generally requires some training. We think that this functionality is important, but that aligning ultrasound with all preoperative data provides real clinical benefit by allowing all modalities to complement each other. 
It is important to note that improving the MRI-ultrasound co-registration accuracy does not automatically imply an improved surgical tool navigation accuracy (TRE). This specific aspect was not tested here and is currently under study. However, since no significant bias was found in our ultrasound calibration and that the error involved in ultrasound probe calibration is generally smaller than the patient-image registration error [77], we hypothesize that improving the MRIultrasound alignment in our system should improve the TRE.

Future developments

New features are currently being developed to address the surgeons' needs and system weaknesses. For example, at 5-10 min, the ultrasound volume reconstruction time is long. A faster, almost real-time technique would facilitate its use. In an upcoming larger series of brain tumor cases, we will now use a new robust rigid body MRI-ultrasound registration technique developed in our laboratory (paper submitted to Medical Image Analysis). Finally, to further improve the system, uncertainty visualization would be a helpful addition by either visualizing the uncertainty in the pointer's tip position as proposed in Wiles et al. [78] or to visualize the uncertainty associated with the segmentation and modeling of different anatomical or functional structures as in Weiler et al. [79].

\section{Conclusions}

Publicly available source code

MincView is a program that includes all the functionalities of IBIS NeuroNav except the ones requiring tool tracking (i.e., it cannot be used for ultrasound image acquisition or probe calibration). It can be used for preoperative surgical planning with advanced data visualization and surface rendering. Its source code is now publicly available from http://www.bic. mni.mcgill.ca/ServicesSoftwareVisualization/MincView.

The aim of this report is to describe and validate a new prototype neuronavigation system that has been developed in our research laboratory over the past decade. The system allows planning and navigation on preoperative images as well as navigation with intraoperative tracked ultrasound. Reconstructing ultrasound in 3D is also possible. Parts of the system's source code are now publicly available on-line. The system was tested intraoperatively in a series of adult brain tumor cases and it was found to have a globally similar accuracy to other similar systems in the literature. The system was useful during surgery, especially because of its ability to improve the MRI-ultrasound alignment at the beginning of surgery. In order to properly compare the ultrasound taken on the dura with the preoperative MRI, the surgeons found that a correction to the registration was needed in five out of nine cases, which shows the importance of such a feature.

Acknowledgments We would like to thank: Dr Vladimir Fonov for writing numerous scripts used for this work; Manny Podaras for helping with the skin fiducials selection; Dr Pierrick Coupe for his useful technical feedback; Dr Rupert Brooks for the design of the ultrasound probe adapter; Dr Micheal Petrides for the fMRI data and Dr Denise Klein for the PET data used in Fig. 4. Funding for this work came from: the Canadian Institutes of Health Research (MOP-74725), the Canadian Natural Science and Engineering Research Council (RGPIN 238739-06), $\mathrm{PhD}$ scholarships from Fond de recherche sur la nature, the Franco Di Giovanni fund, the Alex Pavanel Family fund, the Goals for Lily fund, the Tony Boeckh Brain Tumour Research Fund, the Tony Colannino and Maggie De Fontes Foundation (Brainstorm) and from the Montreal English School Board.

\section{References}

1. Dussik K, Dussik F, Wyt L (1947) Auf dem Wege zur Hyperphonographie des Gehirnes. Wien med Wschr 97:425-429

2. French LA, Wild JJ, Neal D (1950) Detection of cerebral tumors by ultrasonic pulses; pilot studies on postmortem material. Cancer 3:705-708

3. Ballantine HT Jr, Bolt RH, Hueter TF, Ludwig GD (1950) On the detection of intracranial pathology by ultrasound, vol 112. Science, New York, pp 525-528

4. Ballantine HT, Jr, Ludwig GD, Bolt RH, Hueter TF (1950) Ultrasonic localization of the cerebral ventricles. Trans Am Neurol Assoc 51:38-41

5. Voorhies RM, Patterson RH (1980) Preliminary experience with intra-operative ultrasonographic localization of brain tumors. Radiol Nucl Med 10:8-9

6. Rubin JM, Mirfakhraee M, Duda EE, Dohrmann GJ, Brown F (1980) Intraoperative ultrasound examination of the brain. Radiology 137:831-832

7. Iseki $H$, Kawamura $H$, Tanikawa $T$, Kawabatake $H$, Taira $T$, Takakura K, Dohi T, Hata N (1994) An image-guided stereotactic system for neurosurgical operations. Stereotact Funct Neurosurg 63:130-138

8. Koivukangas J, Ylitalo J, Alasaarela E, Tauriainen A (1986) Three-dimensional ultrasound imaging of brain for neurosurgery. Ann Clin Res 18(47):65-72

9. Koivukangas J, Louhisalmi Y, Alakuijala J, Oikarinen J (1993) Ultrasound-controlled neuronavigator-guided brain surgery. J Neurosurg 79:36-42

10. Trobaugh JW, Richard WD, Smith KR, Bucholz RD (1994) Frameless stereotactic ultrasonography: method and applications. Comput Med Imaging Graph 18:235-246

11. Oikarinen J, Jyrki A, Louhisalmi Y, Sallinen S, Helminen H, Koivukangas J (1993) The oulu neuronavigator system: intraoperative ultrasonography in the verification of neurosurgical localization and visualization. In: Maciunas RJ (ed) Interactive image-guided neurosurgery. AANS, USA, pp 233-246

12. Tirakotai W, Miller D, Heinze S, Benes L, Bertalanffy H, Sure U (2006) A novel platform for image-guided ultrasound. Neurosurgery 58:710-718; discussion 710-718

13. Gronningsaeter A, Kleven A, Ommedal S, Aarseth TE, Lie T, Lindseth F, Lang $\varnothing$ T, Unsgard G (2000) SonoWand, an ultrasoundbased neuronavigation system. Neurosurgery 47:1373-1379; discussion 1379-1380 
14. Trantakis C, Meixensberger J, Lindner D, Strauss G, Grunst G, Schmidtgen A, Arnold S (2002) Iterative neuronavigation using 3D ultrasound. A feasibility study. Neurol Res 24:666-670

15. Engelhardt M, Hansen C, Brendel B, Hold S, Brenke C, Pechlivanis I, Harders A, Ermert H, Schmieder K (2007) Real time neuronavigation using 3-D ultrasound and MRI in patients with brain tumor. Advances in medical engineering. Springer, Berlin, pp 59-63

16. Hata N, Dohi T, Iseki H, Takakura K (1997) Development of a frameless and armless stereotactic neuronavigation system with ultrasonographic registration. Neurosurgery 41:608-613; discussion 613-614

17. Tronnier VM, Bonsanto MM, Staubert A, Knauth M, Kunze S, Wirtz CR (2001) Comparison of intraoperative MR imaging and $3 \mathrm{D}$-navigated ultrasonography in the detection and resection control of lesions. Neurosurg Focus 10:1-5

18. Gerganov VM, Akbarian A, Samii A, Samii M, Fahlbusch R (2008) Intraoperative visualization of tumor resection in patients with intracranial tumors - a comparison of two-dimensional ultrasound and high-field MRI. 59th annual meeting of the German society of neurosurgery (DGNC), Würzburg

19. Albert FK, Forsting M, Sartor K, Adams HP, Kunze S (1994) Early postoperative magnetic resonance imaging after resection of malignant glioma: objective evaluation of residual tumor and its influence on regrowth and prognosis. Neurosurgery 34:45-60; discussion $60-61$

20. Unsgaard G, Ommedal S, Muller T, Gronningsaeter A, Nagelhus Hernes TA (2002) Neuronavigation by intraoperative three-dimensional ultrasound: initial experience during brain tumor resection. Neurosurgery 50:804-812; discussion 812

21. Knauth M, Wirtz CR, Tronnier VM, Aras N, Kunze S, Sartor K (1999) Intraoperative MR imaging increases the extent of tumor resection in patients with high-grade gliomas. AJNR Am J Neuroradiol 20:1642-1646

22. Hatiboglu MA, Weinberg JS, Suki D, Rao G, Prabhu SS, Shah K, Jackson E, Sawaya R (2009) Impact of intraoperative high-field magnetic resonance imaging guidance on glioma surgery: a prospective volumetric analysis. Neurosurgery 64:10731081

23. van Velthoven V (2003) Intraoperative ultrasound imaging: comparison of pathomorphological findings in US versus CT, MRI and intraoperative findings. Acta Neurochir Suppl 85:95-99

24. Unsgaard G, Selbekk T, Brostrup Muller T, Ommedal S, Torp SH, Myhr G, Bang J, Nagelhus Hernes TA (2005) Ability of navigated 3D ultrasound to delineate gliomas and metastases-comparison of image interpretations with histopathology. Acta Neurochir (Wien) 147:1259-1269; discussion 1269

25. Lindseth F, Ommedal S, Bang J, Unsgaard G, Nagelhus Hernes TA (2001) Image fusion of ultrasound and MRI as an aid for assessing anatomical shifts and improving overview and interpretation in ultrasound guided neurosurgery. CARS 2001:247-252

26. Nabavi A, Black PM, Gering DT, Westin CF, Mehta V, Pergolizzi RS Jr, Ferrant M, Warfield SK, Hata N, Schwartz RB, Wells WM 3rd, Kikinis R, Jolesz FA (2001) Serial intraoperative magnetic resonance imaging of brain shift. Neurosurgery 48:787-797; discussion 797-798

27. Clatz O, Delingette H, Talos IF, Golby AJ, Kikinis R, Jolesz FA, Ayache N, Warfield SK (2005) Robust nonrigid registration to capture brain shift from intraoperative MRI. IEEE Trans Med Imaging 24:1417-1427

28. Hartkens T, Hill DL, Castellano-Smith AD, Hawkes DJ, Maurer CR Jr, Martin AJ, Hall WA, Liu H, Truwit CL (2003) Measurement and analysis of brain deformation during neurosurgery. IEEE Trans Med Imaging 22:82-92

29. Maurer CRJr, Hill DLG, Martin AJ, Liu H, McCue M, Rueckert D, Lloret D, Hall WA, Maxwell RE, Hawkes DJ, Truwit CL (1998) Investigation of intraoperative brain deformation using a 1.5T interventional MR system: preliminary results. IEEE Trans Med Imaging 17:817-825

30. Hastreiter P, Rezk-Salama C, Soza G, Bauer M, Greiner G, Fahlbusch R, Ganslandt O, Nimsky C (2004) Strategies for brain shift evaluation. Med Image Anal 8:447-464

31. Trantakis C, Tittgemeyer M, Schneider JP, Lindner D, Winkler D, Strauss G, Meixensberger J (2003) Investigation of time-dependency of intracranial brain shift and its relation to the extent of tumor removal using intra-operative MRI. Neurol Res 25:9-12

32. Comeau RM, Fenster A, Peters TM (1998) Intraoperative US in interactive image-guided neurosurgery. Radiographics 18: 1019-1027

33. Schroeder W, Martin K, Lorensen B (2003) The visualization toolkit an object oriented approach to 3D graphics, VTK version 4.2. 3, vol 4. Kitware, New York

34. Neelin P (1998) The MINC file format: from bytes to brains. NeuroImage 7:786

35. Gobbi DG, Comeau RM, Peters TM (1999) Ultrasound probe tracking for real-time ultrasound/MRI overlay and visualization of brain shift. MICCAI 1999, vol 1679. Lecture Notes in Computer Science. Springer, Cambridge, UK, pp 920-927

36. Mercier L, Lango T, Lindseth F, Collins DL (2005) A review of calibration techniques for freehand 3-D ultrasound systems. Ultrasound Med Biol 31:449-471

37. Treece GM, Gee AH, Prager RW, Cash CJ, Berman LH (2003) High-definition freehand 3-D ultrasound. Ultrasound Med Biol 29:529-546

38. Prager RW, Gee A, Berman L (1998) Stradx: real-time acquisition and visualization of freehand three-dimensional ultrasound. Med Image Anal 3:129-140

39. Lindseth F, Tangen GA, Lang $\varnothing$ T, Bang J (2003) Probe calibration for freehand 3-D ultrasound. Ultrasound Med Biol 29:16071623

40. Blackall JM, Rueckert D, Maurer CR, Penny GP, Hill DLG, Hawkes DJ (2000) An image registration approach to automated calibration for freehand 3D ultrasound. MICCAI 2000, vol 1935. Lecture Notes in Computer Science. Springer, Pittsburgh, USA, pp 462-471

41. Maurer CRJr, Fitzpatrick JM, Wang MY, Galloway RLJr, Maciunas RJ, Allen GS (1997) Registration of head volume images using implantable fiducial markers. IEEE Trans Med Imaging 16: $447-462$

42. Fitzpatrick JM, West JB, Maurer CR Jr (1998) Predicting error in rigid-body point-based registration. IEEE Trans Med Imaging 17:694-702

43. Jannin P, Fitzpatrick JM, Hawkes DJ, Pennec X, Shahidi R, Vannier MW (2002) Validation of medical image processing in image-guided therapy. IEEE Trans Med Imaging 21:1445-1449

44. Comeau RM, Sadikot AF, Fenster A, Peters TM (2000) Intraoperative ultrasound for guidance and tissue shift correction in imageguided neurosurgery. Med Phys 27:787-800

45. Arbel T, Morandi X, Comeau RM, Collins DL (2001) Automatic non-linear MRI-ultrasound registration for the correction of intraoperative brain deformations. MICCAI 2001, vol 2208. LNCS. Springer, Utrecht, The Netherlands, pp 913-922

46. Arbel T, Morandi X, Comeau RM, Collins DL (2004) Automatic non-linear MRI-ultrasound registration for the correction of intraoperative brain deformations. Comput Aided Surg 9:123-136

47. Klein D, Olivier A, Milner B, Zatorre RJ, Johnsrude I, Meyer E, Evans AC (1997) Obligatory role of the LIFG in synonym generation: evidence from PET and cortical stimulation. Neuroreport 8:3275-3279

48. Amiez C, Kostopoulos P, Champod AS, Collins DL, Doyon J, Del Maestro R, Petrides M (2008) Preoperative functional magnetic resonance imaging assessment of higher-order cognitive func- 
tion in patients undergoing surgery for brain tumors. J Neurosurg 108:258-268

49. Sled JG, Zijdenbos AP, Evans AC (1998) A nonparametric method for automatic correction of intensity nonuniformity in MRI data. IEEE Trans Med Imaging 17:87-97

50. Collins DL, Neelin P, Peters TM, Evans AC (1994) Automatic 3D intersubject registration of MR volumetric data in standardized Talairach space. J Comput Assis Tomogr 18:192-205

51. Zijdenbos AP, Dawant BM, Margolin RA, Palmer AC (1994) Morphometric analysis of white matter lesions in MR images: method and validation. IEEE Trans Med Imaging 13:716-724

52. MacDonald D, Kabani N, Avis D, Evans AC (2000) Automated 3-D extraction of inner and outer surfaces of cerebral cortex from MRI. Neuroimage 12:340-356

53. Bucholz RD, Yeh DD, Trobaugh JW, McDurmott LL (1997) The correction of stereotactic inaccuracy caused by brain shift using an intraoperative ultrasound device. CVRMed MRCAS'97, pp 459-466

54. Solberg OV, Lindseth F, Torp H, Blake RE, Nagelhus Hernes TA (2007) Freehand 3D ultrasound reconstruction algorithms-a review. Ultrasound Med Biol 33:991-1009

55. Coupe P, Hellier P, Morandi X, Barillot C (2007) A probabilistic objective function for $3 \mathrm{~d}$ rigid registration of intraoperative us and preoperative MR brain images. ISBI, Arlington 1320-1323

56. Ji S, Wu Z, Hartov A, Roberts DW, Paulsen KD (2008) Mutualinformation-based image to patient re-registration using intraoperative ultrasound in image-guided neurosurgery. Med Phys 35:4612-4624

57. Reinertsen I, Descoteaux M, Drouin S, Siddiqi K, Collins DL (2004) Vessel driven correction of brain shift. MICCAI 2004, vol 3217, LNCS. Springer, Rennes, France, pp 208-216

58. Reinertsen I, Lindseth F, Unsgaard G, Collins DL (2007) Clinical validation of vessel-based registration for correction of brain-shift. Med Image Anal 11:673-684

59. Collignon A, Maes F, Delaere D, Vandermeulen D, Suetens P, Marchal G (1995) Automated multi-modality image registration based on information theory. IPMI'95 3:263-274

60. Lindner D, Trantakis C, Renner C, Arnold S, Schmitgen A, Schneider J, Meixensberger J (2006) Application of intraoperative 3D ultrasound during navigated tumor resection. Minim Invasive Neurosurg 49:197-202

61. Miller D, Heinze S, Tirakotai W, Bozinov O, Surucu O, Benes L, Bertalanffy H, Sure U (2007) Is the image guidance of ultrasonography beneficial for neurosurgical routine? Surg Neurol 67: 579-587; discussion 587-588

62. Prager RW, Rohling RN, Gee AH, Berman L (1998) Rapid calibration for 3-D freehand ultrasound. Ultrasound Med Biol 24:855-869

63. Kowal J, Amstutz CA, Caversaccio M, Nolte L-P (2003) On the development and comparative evaluation of an ultrasuond B-mode probe calibration method. Comput Aided Surg 8:107-119
64. Rousseau F, Hellier P, Barillot C (2006) A novel temporal calibration method for 3-D ultrasound. IEEE Trans Med Imaging 25:1108-1112

65. Mascott CR, Sol JC, Bousquet P, Lagarrigue J, Lazorthes Y, Lauwers-Cances V (2006) Quantification of true in vivo (application) accuracy in cranial image-guided surgery: influence of mode of patient registration. Neurosurgery 59:146-155

66. Pfisterer WK, Papadopoulos S, Drumm DA, Smith K, Preul MC (2008) Fiducial versus nonfiducial neuronavigation registration assessment and considerations of accuracy. Neurosurgery 62 : 201-207; discussion 207-208

67. Villalobos H, Germano IM (1999) Clinical evaluation of multimodality registration in frameless stereotaxy. Comput Aided Surg $4: 45-49$

68. Woerdeman PA, Willems PWA, Noordmans HJ, Berkelbachvander Sprenkel JW (2006) Clinical accuracy of neuronavigation using registration methods based on point-pairs or surface matching. Int J CARS 1:297-298

69. Dorward NL, Alberti O, Palmer JD, Kitchen ND, Thomas DG (1999) Accuracy of true frameless stereotaxy: in vivo measurement and laboratory phantom studies. Technical note. J Neurosurg 90:160-168

70. Shamir RR, Joskowicz L, Spektor S, Shoshan Y (2008) Localization and registration accuracy in image-guided neurosurgery: a clinical study. Int J CARS 3:S289

71. Fitzpatrick JM (2009) Fiducial registration error and target registration error are uncorrelated. SPIE Med Imaging 2009:7261

72. Shamir RR, Joskowicz L (2009) Worst-case analysis of target localization errors in fiducial-based rigid body registration. In: Josien PWP, Benoit MD (eds) vol 7259. SPIE 725938

73. Fitzpatrick JM (2010) The role of registration in accurate surgical guidance. J Eng Med 224:607-622

74. Letteboer MM, Willems PW, Viergever MA, Niessen WJ (2005) Brain shift estimation in image-guided neurosurgery using 3-D ultrasound. IEEE Trans Biomed Eng 52:268-276

75. Keles GE, Lamborn KR, Berger MS (2003) Coregistration accuracy and detection of brain shift using intraoperative sononavigation during resection of hemispheric tumors. Neurosurgery 53:556-562; discussion 562-564

76. Ji S, Liu F, Hartov A, Roberts DW, Paulsen KD (2007) Brain-skull boundary conditions in a computational deformation model. In: Proceedings of SPIE, vol 6509, San Diego, USA 65092J

77. Lindseth F, Bang J, Lang $\varnothing \mathrm{T}$ (2003) A robust and automatic method for evaluating accuracy in 3-D ultrasound-based navigation. Ultrasound Med Biol 29:1439-1452

78. Wiles A, Peters T (2008) Interactive software tool for IGS performance evaluation. Int J CARS 3:S314

79. Weiler F, Hahn HK, Koehn A, Friman O, Klein J, Peitgen $\mathrm{H}-\mathrm{O}$ (2008) Dealing with inaccuracies in multimodal neurosurgical planning - a preliminary concept. Int J CARS 3:S77-S78 